\title{
$A b$ initio multi-reference perturbation theory calculations of the ground and low-lying electronic states of the KRb molecule
}

\author{
M.B. Shundalau ${ }^{a}{ }^{*}$, G.A. Pitsevich ${ }^{a}$, A.E. Malevich ${ }^{a}$, A.V. Hlinisty ${ }^{a}$, A.A. Minko ${ }^{a}$, R. Ferber ${ }^{b}$, M. Tamanis $^{b}$ \\ ${ }^{a}$ Physics Department, Belarusian State University, 4 Nezalezhnasti Ave., 220030 Minsk, Belarus \\ ${ }^{\mathrm{b}}$ Laser Centre of the University of Latvia, Zellu Str. 8, LV-1002 Riga, Latvia
}

\section{A R T I C L E I N F O}

\section{Article history:}

Received 6 April 2016

Accepted 28 April 2016

Available online 4 May 2016

\section{Keywords:}

Multi-reference perturbation theory

calculations

KRb molecule

Potential energy curves

Vibrational states

Franck-Condon factors

\begin{abstract}
A B S T R A C T
The potential energy curves of the low-lying electronic states correlating up to the limit $K(4 p)+R b(5 s)$ of $\mathrm{KRb}$ molecule have been calculated using the multi-reference perturbation theory method at the CASSCF/ XMCQDPT2 level of theory without and with spin-orbit coupling. The calculated parameters of the ground $X^{1} \Sigma^{+}$state are in the best agreement among all previously performed $a b$ initio calculations for the $\mathrm{KRb}$ molecule. The calculated vibrational intervals of the ground electronic term of the ${ }^{39} \mathrm{~K}^{85} \mathrm{Rb}$ molecule describe the experiment with the accuracy within $\pm 1 \mathrm{~cm}^{-1}$. The calculated intensities of the $2^{1} \Sigma^{+}$ $\left(v^{\prime}=3, J^{\prime}=26\right) \rightarrow X^{1} \Sigma^{+}\left(v^{\prime \prime}=0 \ldots 24, J^{\prime \prime}=25,27\right)$ transitions satisfactory reflect the experimentally observed intensities distribution.
\end{abstract}

(c) 2016 Elsevier B.V. All rights reserved.

\section{Introduction}

The $\mathrm{KRb}$ molecule is among the most intensively studied polar alkali diatomics, partially because of its successful usage in production and investigation in cold and ultracold conditions (see for instance [1-4]). Recent efforts are often focused on transferring weakly bound ultracold molecules into their "absolute" ground state $X\left(v^{\prime \prime}=0, J^{\prime \prime}=0\right)$ by additional optical cycles via excited electronic states. The efficiency of these processes depends rather critically on the proper choice of optical paths, which needs reliable information about the potential energy curves (PECs) of the combining electronic states in wide range of energies and internuclear distances. Since the empirical information based on spectroscopy studies is still insufficient, the analysis of the efficiency of optical paths has to include the data from $a b$ initio calculations (see for KRb the recent paper [5]).

The goal of the present paper is to perform $a b$ initio calculations of PECs of the ground and several excited electronic states of the $\mathrm{KRb}$ molecule corresponding to the first three asymtotic limits of separated atoms and to derive molecular constants, vibrationalrotation energies and Franck-Condon factors. The calculation of the electronic structure of molecular systems, which contain a heavy alkali metals atom, is a non-trivial task that requires taking into account the relativistic effects for the core electrons, the static

\footnotetext{
* Corresponding author.

E-mail address: shundalov@bsu.by (M.B. Shundalau).
}

and dynamic components of the correlation energy, a large number of excited configurations, etc. The diatomic polar molecule $\mathrm{KRb}$ is a typical example of the above-mentioned systems. The electronic states of ${ }^{39} \mathrm{~K}^{85} \mathrm{Rb}$ (the most abundant isopotomer, $67.3 \%$ ) and others stable isopotomers of the KRb molecule $\left({ }^{39} \mathrm{~K}^{87} \mathrm{Rb}, 26.0 \%\right.$, ${ }^{41} \mathrm{~K}^{85} \mathrm{Rb}, 4.9 \%,{ }^{41} \mathrm{~K}^{87} \mathrm{Rb}, 1.9 \%$ ) were studied experimentally in [14,6-29] and theoretically in [5,30-44]. The investigation of the ground state $X^{1} \Sigma^{+}$was performed by Ross et al. [6], Amiot and Vergès [9], Stwalley [20], Wang et al. [22] and Pashov et al. [13] by employing high-resolution spectra of laser-induced fluorescence yielding the highly accurate PEC. Regarding the excited states, fragmentary experimental data for the next $\left(2^{1} \Sigma^{+}\right)$singlet state are available in $[4,6]$. The Dunham coefficients and RKR potential of the $3^{1} \Sigma^{+}$state can be found in Amiot et al. [8]. Aikawa et al. [26] obtained the parameters of the $3^{1} \Sigma^{+}$state PEC. Molecular constants of the "twin" $1^{1} \Pi$ and $2^{1} \Pi$ states have been obtained by Kasahara et al. [7], Amiot et al. [10] and Okada et al. [16]; note that the molecular constants of the $2^{1} \Pi$ state obtained in $[7,10]$ differ substantially. Low-lying KRb triplet terms have been investigated by Pashov et al. [13] $\left(1^{3} \Sigma^{+}\right)$, Kobayashi et al. [15] ( $\left.1^{3} \Pi\right)$, Amiot [11] $\left(2^{3} \Sigma^{+}\right)$, Lee et al. [12,17], Kim et al. [23] $\left(3^{3} \Sigma^{+}\right)$, Banerjee et al. [14] $\left(2^{3} \Sigma^{+}, 1^{1} \Pi\right)$ and Kim et al. [23] ( $\left.2^{3} \Pi\right)$. Highly-excited states of the $\mathrm{KRb}$ molecule were studied in $[8,12,21,23,25,27,29,43]$.

$\mathrm{Ab}$ initio calculations of the $\mathrm{KRb}$ electronic states are traditionally [30-34,40,41,43] performed in the multi-configuration CASSCF/MRCI (Complete Active Space Self-Consistent Field/Multi- 
Reference Configuration Interaction) approximation. Such an approach in the case of a multi-configuration problem allows one considering both static part of correlation energy (at the CASSCF level of theory) and its dynamic component (at the MRCI level of theory). Note that both levels of theory, which are consistently applied here, are based on a variational principle. The common feature of calculations $[32,33,41]$ is the sufficiently successful reproduction of the experimental value of the equilibrium internuclear distance $R_{e}$ equal to $4.06770 \AA$ [13] of the ground electronic state $X^{1} \Sigma^{+}$, namely 4.055 [32], 4.09 [33] and $4.095 \AA$ [41]. At the same time these calculations yield significantly lower values of the ground state dissociation energy $D_{e}$, namely 4110 [32], 4113 [33] and $4128.8 \mathrm{~cm}^{-1}$ [41] if compared with the experimental value $4217.815 \mathrm{~cm}^{-1}$ [13]. Meyer and Bohn [40] at the abovementioned level of theory received $R_{e}=4.33 \AA$ and $D_{e}=4039 \mathrm{~cm}^{-1}$. At the coupled clusters (MRCC) level of theory Meyer and Bohn [40] and Fedorov et al. [44] received significantly lower $\left(4062 \mathrm{~cm}^{-1}\right)$ and higher $\left(4306 \mathrm{~cm}^{-1}\right)$ values of $D_{e}$, respectively.

Recently [45] a new approach to the MS-MR-PT (Multi-State Multi-Reference Perturbation Theory) level of theory has been developed, which is called XMCQDPT (Extended MultiConfiguration Quasi-Degenerate Perturbation Theory). This approach allows one to look at the considered problem from an alternative point of view, which is not based on variational principle, but exploits the perturbation theory methods. In particular, the XMCQDPT2 method proposed in [45] is approximately sizeconsistent for low-lying states in contrast to MRCI and other CI methods, which are not size-consistent.

The main goals of this work are: (i) to calculate the PECs of the ground and some low-lying excited states of the $\mathrm{KRb}$ molecule at the multi-reference perturbation level of theory and (ii) basing only on the performed without any fitting $a b$ initio PECs calculations, to determine other spectroscopic characteristics of the states (vibrational and vibration-rotational energies, etc.). The main attention is paid to the ground $X^{1} \Sigma^{+}$and first triplet $1^{3} \Sigma^{+}$states of the KRb molecule. The empirical PECs of these states are determined with a high accuracy by Pashov et al. [13]. It allows using these PECs as a kind of benchmark for the $a b$ initio calculations at the high level of theory. The quality of the present calculated PECs was tested by comparing the predicted Franck-Condon factors with the relative intensity distribution in the $2^{1} \Sigma^{+} \rightarrow X^{1} \Sigma^{+}$ transition measured in Laser Centre, University of Latvia.

\section{Computations}

The small core Stuttgart RSC ECP [46] has been used in our calculations. The Gaussian basis sets used are $(11 \mathrm{~s} 11 \mathrm{p} 5 \mathrm{~d} 3 \mathrm{f}) \rightarrow[8 \mathrm{~s} 8 \mathrm{p} 5 \mathrm{~d} 3 \mathrm{f}]$ $(\mathrm{ECP} 10 \mathrm{MDF})$ for the potassium atom and $(13 \mathrm{~s} 10 \mathrm{p} 5 \mathrm{~d} 3 \mathrm{f}) \rightarrow$ [8s7p5d3f] (ECP28MDF) for the rubidium atom [46].

First, the CASSCF calculations with the state-averaged (SA) procedure were done. The active space for the CASSCF calculations was 2 electrons in 14 orbitals. The SA procedure was realized for singlet and triplet states. The $\operatorname{SA}-\operatorname{CASSCF}(2,14)$ calculations were performed pointwisely with the step of $0.01 \AA$ in the range of interatomic distances from 3.00 to $6.00 \AA$. In contrast to the automatic PEC scanning, which is less time consuming, but often leads to a divergence of solution at a large distance from the starting point, the point-by-point calculations allow to get the convergence of the solution at the CASSCF level of theory at each point under consideration, as well as to avoid "jumps" of energy and PEC's breaks. A lack of convergence at using the automatic PEC's scanning can lead to the errors in the calculations of PEC's shape [41] (see below). Outside the specified range (2.50-3.00, 6.00-15.00 and 15.00-20.00 $\AA$ ) the calculations were performed with the steps of
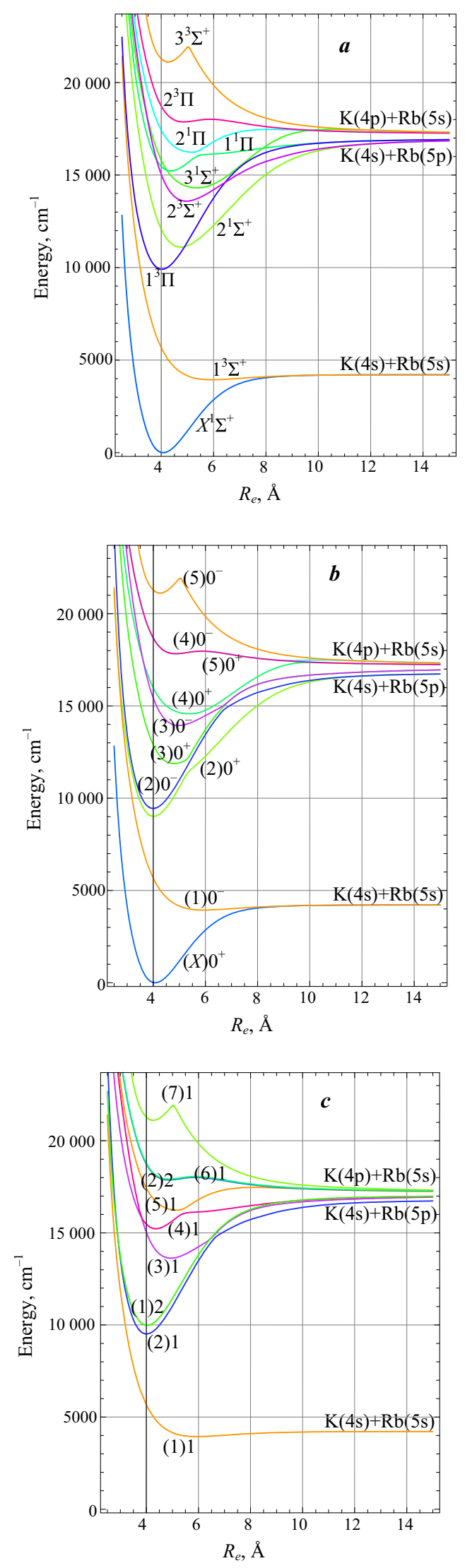

Fig. 1. Calculated at the CASSCF(2,14)/XMCQDPT2 level of theory low-lying singlet and triplet (a) and spin-mixed with $\Omega=0^{+}, 0^{-}$(b) and $\Omega=1,2(c)$ terms of the KRb molecule. 
Table 1

Molecular spectroscopic constants of ${ }^{39} \mathrm{~K}^{85} \mathrm{Rb}$ molecule.

\begin{tabular}{|c|c|c|c|c|c|c|c|c|}
\hline \multirow[t]{2}{*}{ Term } & \multicolumn{2}{|l|}{$T_{e}, \mathrm{~cm}^{-1}$} & \multicolumn{2}{|l|}{$R_{e}, \AA$} & \multicolumn{2}{|l|}{$D_{e}, \mathrm{~cm}^{-1}$} & \multicolumn{2}{|l|}{$\omega_{e}, \mathrm{~cm}^{-1}$} \\
\hline & Experimental & Calculated & Experimental & Calculated & Experimental & Calculated & Experimental & Calculated \\
\hline$X^{1} \Sigma^{+}$ & 0.0 & 0.0 & $4.06770^{\mathrm{a}}$ & $\begin{array}{l}4.055^{\mathrm{b}} \\
4.09^{\mathrm{c}} \\
4.33^{\mathrm{d}} \\
4.05^{\mathrm{d}} \\
4.095^{\mathrm{e}} \\
4.073^{\mathrm{f}} \\
4.076^{\mathrm{g}}\end{array}$ & $\begin{array}{l}4217.815^{\mathrm{a}} \\
4217.91^{\mathrm{h}} \\
4217.822^{\mathrm{i}}\end{array}$ & $\begin{array}{l}4110^{\mathrm{b}} \\
4113^{\mathrm{c}} \\
4039^{\mathrm{d}} \\
4062^{\mathrm{d}} \\
4129^{\mathrm{e}} \\
4306^{\mathrm{f}} \\
4213^{\mathrm{g}}\end{array}$ & $\begin{array}{l}75.8713^{\mathrm{a}} \\
75.8458^{\mathrm{j}}\end{array}$ & $\begin{array}{l}76.3^{\mathrm{b}} \\
76^{\mathrm{c}} \\
75.395^{\mathrm{e}} \\
75.3^{\mathrm{f}} \\
75.232^{\mathrm{g}}\end{array}$ \\
\hline $1^{3} \Sigma^{+}$ & $3968.784^{\mathrm{a}}$ & $\begin{array}{l}3871^{\mathrm{b}} \\
3845^{\mathrm{c}} \\
3901^{\mathrm{e}} \\
3938^{\mathrm{g}}\end{array}$ & $5.9029^{\mathrm{a}}$ & $\begin{array}{l}5.901^{\mathrm{b}} \\
5.94^{\mathrm{c}} \\
5.920^{\mathrm{e}} \\
5.952^{\mathrm{g}}\end{array}$ & $249.031^{\mathrm{a}}$ & $\begin{array}{l}239^{\mathrm{b}} \\
242^{\mathrm{c}} \\
238^{\mathrm{e}} \\
275^{\mathrm{g}}\end{array}$ & $17.579^{a}$ & $\begin{array}{l}17.4^{\mathrm{b}} \\
18^{\mathrm{c}} \\
17.931^{\mathrm{e}} \\
19.234^{\mathrm{g}}\end{array}$ \\
\hline $1^{3} \Pi$ & $9720.531^{\mathrm{k}}$ & $\begin{array}{l}9718^{\mathrm{b}} \\
9721^{\mathrm{c}} \\
9911^{\mathrm{e}} \\
9910^{\mathrm{g}}\end{array}$ & $4.02698^{k}$ & $\begin{array}{l}4.023^{\mathrm{b}} \\
4.06^{\mathrm{c}} \\
4.071^{\mathrm{e}} \\
4.024^{\mathrm{g}}\end{array}$ & & $\begin{array}{l}7129^{\mathrm{b}} \\
7017^{\mathrm{c}} \\
6682^{\mathrm{e}} \\
7019^{\mathrm{g}}\end{array}$ & & $\begin{array}{l}77.4^{\mathrm{b}} \\
78^{\mathrm{c}} \\
65.973^{\mathrm{e}} \\
76.929^{\mathrm{g}}\end{array}$ \\
\hline $2^{1} \Sigma^{+}$ & & $\begin{array}{l}10862^{\mathrm{b}} \\
10804^{\mathrm{c}} \\
11104^{\mathrm{g}}\end{array}$ & & $\begin{array}{l}4.707^{b} \\
4.74^{\mathrm{c}} \\
4.747^{\mathrm{g}}\end{array}$ & & $\begin{array}{l}5985^{\mathrm{b}} \\
5968^{\mathrm{c}} \\
5807^{\mathrm{g}}\end{array}$ & & $\begin{array}{l}57.7^{\mathrm{b}} \\
58^{\mathrm{c}} \\
56.639^{\mathrm{g}}\end{array}$ \\
\hline $2^{3} \Sigma^{+}$ & $13507.146^{1}$ & $\begin{array}{l}13423^{b} \\
13323^{c} \\
13588^{g}\end{array}$ & $4.983^{1}$ & $\begin{array}{l}4.953^{b} \\
5.00^{c} \\
4.967^{g}\end{array}$ & & $\begin{array}{l}3424^{b} \\
3388^{c} \\
3323^{g}\end{array}$ & $48.638^{1}$ & $\begin{array}{l}48.6^{\mathrm{b}} \\
49^{\mathrm{c}} \\
51.750^{\mathrm{g}}\end{array}$ \\
\hline $3{ }^{1} \Sigma^{+}$ & $\begin{array}{l}13995.46^{\mathrm{m}} \\
13995.5113^{\mathrm{n}}\end{array}$ & $\begin{array}{l}13985^{\mathrm{b}} \\
13886^{\mathrm{c}} \\
14306^{\mathrm{g}}\end{array}$ & $\begin{array}{l}5.259018^{\mathrm{m}} \\
5.25904119^{\mathrm{n}}\end{array}$ & $\begin{array}{l}5.218^{\mathrm{b}} \\
5.21^{\mathrm{c}} \\
5.359^{\mathrm{g}}\end{array}$ & $3246.0363^{n}$ & $\begin{array}{l}3148^{\mathrm{b}} \\
3226^{\mathrm{c}} \\
2961^{\mathrm{g}}\end{array}$ & $40.763^{\mathrm{m}}$ & $\begin{array}{l}39.9^{b} \\
41^{c} \\
36.106^{g}\end{array}$ \\
\hline $1^{1} \Pi$ & $15012.493^{o, p}$ & $\begin{array}{l}15034^{\mathrm{b}} \\
15011^{\mathrm{c}} \\
15224^{\mathrm{g}}\end{array}$ & $4.3813^{\mathrm{o}, \mathrm{p}}$ & $\begin{array}{l}4.370^{\mathrm{b}} \\
4.40^{\mathrm{c}} \\
4.363^{\mathrm{g}}\end{array}$ & $2021.5^{\circ}$ & $\begin{array}{l}1813^{\mathrm{b}} \\
1774^{\mathrm{c}} \\
1705^{\mathrm{g}}\end{array}$ & $61.256^{o, p}$ & $\begin{array}{l}61.3^{\mathrm{b}} \\
60^{\mathrm{c}} \\
62.884^{\mathrm{g}}\end{array}$ \\
\hline $2^{1} \Pi$ & $\begin{array}{l}16210.252^{\circ} \\
15902.06^{\mathrm{q}}\end{array}$ & $\begin{array}{l}15835^{\mathrm{b}} \\
15777^{\mathrm{c}} \\
16232^{\mathrm{g}}\end{array}$ & $\begin{array}{l}5.0822^{\circ} \\
5.088^{\mathrm{q}}\end{array}$ & $\begin{array}{l}5.048^{\mathrm{b}} \\
5.06^{\mathrm{c}} \\
5.170^{\mathrm{g}}\end{array}$ & & $\begin{array}{l}1298^{\mathrm{b}} \\
1210^{\mathrm{c}} \\
1019^{\mathrm{g}}\end{array}$ & $\begin{array}{l}51.70^{\circ} \\
49.76^{\mathrm{q}}\end{array}$ & $\begin{array}{l}55.4^{b} \\
54^{\mathrm{c}} \\
42.728^{\mathrm{g}}\end{array}$ \\
\hline $2^{3} \Pi_{\Omega}$ & $\begin{array}{l}\Omega=0^{+} / 0^{-}, \text {or }(5) 0^{+} /(4) 0^{-} \\
17369.8^{\mathrm{r}}\end{array}$ & $\begin{array}{l}17303^{\mathrm{b}} \\
17245^{\mathrm{c}} \\
17828^{\mathrm{g}}\end{array}$ & & $\begin{array}{l}4.763^{b} \\
4.91^{\mathrm{c}} \\
4.856^{\mathrm{g}}\end{array}$ & & & $39.4^{\mathrm{r}}$ & $38^{c}$ \\
\hline & $\begin{array}{l}\Omega=1, \text { or }(6) 1 \\
17408.8^{\mathrm{r}}\end{array}$ & $\begin{array}{l}17323^{\mathrm{b}} \\
17274^{\mathrm{c}} \\
17872^{\mathrm{g}}\end{array}$ & & $\begin{array}{l}4.868^{\mathrm{b}} \\
4.91^{\mathrm{c}} \\
4.855^{\mathrm{g}}\end{array}$ & & & $37.7^{\mathrm{r}}$ & $40^{c}$ \\
\hline & $\begin{array}{l}\Omega=2, \text { or }(2) 2 \\
17441.8^{\mathrm{r}}\end{array}$ & $\begin{array}{l}17339^{\mathrm{b}} \\
17296^{\mathrm{c}} \\
17914^{\mathrm{g}}\end{array}$ & & $\begin{array}{l}4.868^{b} \\
4.92^{\mathrm{c}} \\
4.855^{\mathrm{g}}\end{array}$ & & & $39.0^{\mathrm{r}}$ & $40^{c}$ \\
\hline $3^{3} \Sigma^{+}$ & $\begin{array}{l}18601.322^{\mathrm{s}} \\
18601.25^{\mathrm{t}}\end{array}$ & $\begin{array}{l}18524^{b} \\
18528^{\mathrm{c}} \\
21109^{\mathrm{g}}\end{array}$ & $4.4121^{t}$ & $\begin{array}{l}4.445^{\mathrm{b}} \\
4.44^{\mathrm{c}} \\
4.264^{\mathrm{g}}\end{array}$ & & & $\begin{array}{l}64.530^{\mathrm{s}} \\
64.56^{\mathrm{t}}\end{array}$ & $65^{c}$ \\
\hline
\end{tabular}

Notes:

a [13].

b $[32]$.

c [33].

d $[40]$.

e [41].

f [44].

$\mathrm{g}$ This work.

h $[20]$.

i [22].

j [9].

$\mathrm{k}$ [15].

1 [11].

m $[8]$.

n [26].

o [7].

p [16].

q [10].

r [23].

s [12].

$\mathrm{t}$ [17].

0.1 and $0.25 \AA$, respectively. Then the calculations for singlet and triplet states were performed in the XMCQDPT2 [45] approximation. All eight lowest double occupied orbitals were included in the perturbation based calculations. The ISA shift of $0.20[47,48]$ was used in present approach. The dimension of the effective Hamiltonian was $13 \times 13$ [48]. Finally the spin-orbit coupling (SOC) calculations were performed with the one-electron PauliBreit operator. All these calculations were performed using a 


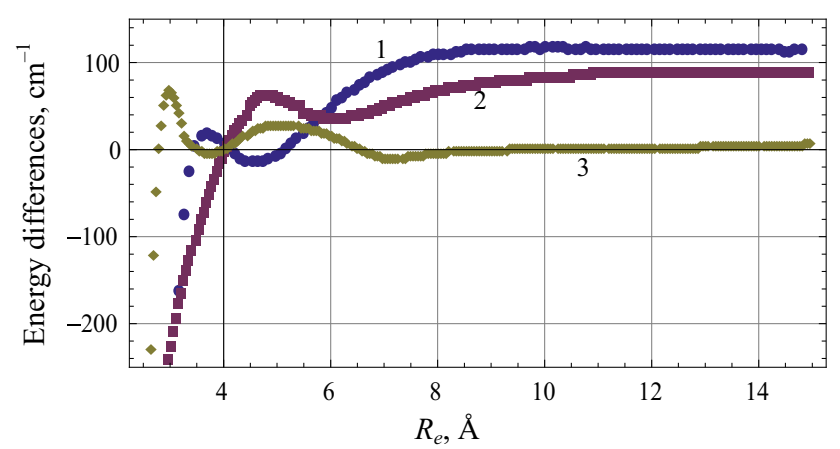

Fig. 2. Energy differences between empirical PEC [13] ( $\left.E_{\text {empir }}\right)$ and ab initio PECs $\left(E_{\text {calc }}\right)$ for the $\mathrm{KRb}$ ground state $X^{1} \Sigma^{+}$term: (1) Rousseau et al. [32], (2) Chen et al. [41], (3) present work.

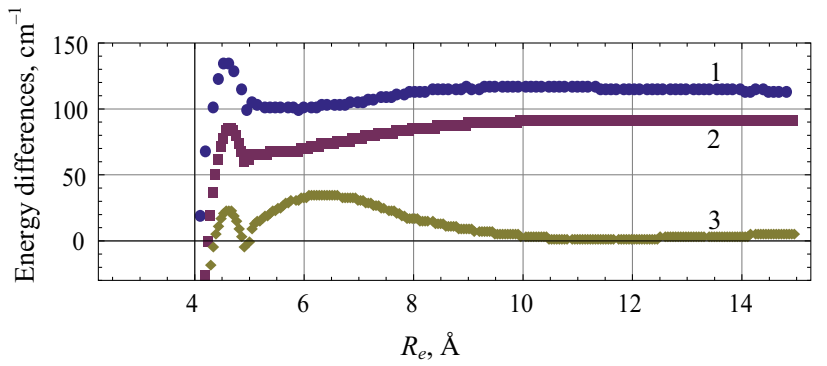

Fig. 3. Energy differences between empirical PEC [13] ( $\left.E_{\text {empir }}\right)$ and $a b$ initio PECs $\left(E_{\text {calc }}\right)$ with respect to the bottom of the ground singlet state for the KRb first triplet state $1^{3} \Sigma^{+}$term: (1) Rousseau et al. [32], (2) Chen et al. [41], (3) present work.

quantum chemical package Firefly [49]. The electronic energy terms $T_{e}$ and the equilibrium internuclear distances $R_{e}$ were obtained using fourth degree polynomial approximation of the ab initio PECs near their minima.

The calculations of the vibrational states energies were performed by numerical Fourier-transform based solution of the corresponding Schrödinger equation [50,51].

\section{Results and discussion}

\subsection{Electronic states}

The PECs calculations have been performed for the singlet and triplet states (without and with spin-orbit coupling) correspond- ing to the three lowest dissociation limits: $K(4 s)+R b(5 s), K(4 s)$ $+\mathrm{Rb}(5 \mathrm{p})$ and $\mathrm{K}(4 \mathrm{p})+\mathrm{Rb}(5 \mathrm{~s})$. The ground state $X^{1} \Sigma^{+}$and the first triplet state $1^{3} \Sigma^{+}$correspond to the first asymptotic limit, the singlet states $2^{1} \Sigma^{+}, 1^{1} \Pi$ and the triplet states $1^{3} \Pi, 2^{3} \Sigma^{+}$correspond to the second one, and the singlet states $3^{1} \Sigma^{+}, 2^{1} \Pi$ and the triplet states $2^{3} \Pi, 3^{3} \Sigma^{+}$correspond to the third one. When the SOC is taken into account, the $X^{1} \Sigma^{+}, 2^{1} \Sigma^{+}$and $3^{1} \Sigma^{+}$terms correspond to the $(X) 0^{+},(3) 0^{+}$and $(4) 0^{+}$terms, respectively; the $1^{1} \Pi$ and $2^{1} \Pi$ terms correspond to the (4) 1 and (5) 1 terms, respectively; the $1^{3} \Sigma^{+}, 2^{3} \Sigma^{+}$and $3^{3} \Sigma^{+}$terms split into the $(1) 0^{-}$and $(1) 1,(3) 0^{-}$ and (3) 1 and $(5) 0^{-}$and (7) 1 terms, respectively; the $1^{3} \Pi$ and $2^{3} \Pi$ terms split into the $(2) 0^{+},(2) 0^{-},(2) 1$ and (1)2 and $(5) 0^{+},(4) 0^{-}$, (6) 1 and (2)2 terms, respectively.

The calculated PECs versus internuclear distances are shown in Fig. 1 and are also presented in the supplementary material. The calculated molecular spectroscopic constants (the electronic term energy $T_{e}$, the equilibrium internuclear distance $R_{e}$, the dissociation energy $D_{e}$ and the harmonic vibrational frequency $\omega_{e}$ ) are presented and compared with experimental and previous theoretical studies in Table 1.

The calculated molecular constants $R_{e}$ and $D_{e}$ for the ground electronic state $X^{1} \Sigma^{+}\left(R_{e}=4.07581 \AA, D_{e}=4213.1 \mathrm{~cm}^{-1}\right)$ agree considerably well with the experimental data [13] $\left(R_{e}=4.06770 \AA\right.$, $\left.D_{e}=4217.815 \mathrm{~cm}^{-1}\right)$. As can be seen from Table 1 , the $R_{e}$ value and particularly the $D_{e}$ value obtained from present calculations are in better agreement with the experiment than the respective values obtained in previous $a b$ initio calculations.

Besides the above-mentioned spectroscopic parameters, which characterize the behavior of a PEC near the minimum and its asymptotic limit, the PEC's shape in the wide range of internuclear distances is also important. In Fig. 2 the present $X^{1} \Sigma^{+}$state PEC is compared with respective analytical empirical [13] and two $a b$ initio curves $[32,41]$. The energy differences between the empirical and calculated curves are presented with respect to the bottom of the ground state potential well. These data indicate that the present calculated ground state PEC (see curve 3 ) agree better with the empirical PEC [13]. Almost throughout the range of $R$ above $3.25 \AA$ the deviations of the calculated energies from the empirical ones do not exceed $30 \mathrm{~cm}^{-1}$; especially good agreement is observed in the range of $R$ above $6.0 \AA$. An exception is the region near $3 \AA$. It is worth mentioning that this is the internuclear distance value at which the empirical PEC is matched with its asymptotic short range part.

The energy difference $E_{\text {empir }}-E_{\text {calc }}$ for the calculation by Chen et al. [41], see curve 2 in Fig. 2, demonstrates "jumps" by approximately $13,-6$ and $2 \mathrm{~cm}^{-1}$ in the regions of $4.45-4.50,5.45-5.50$

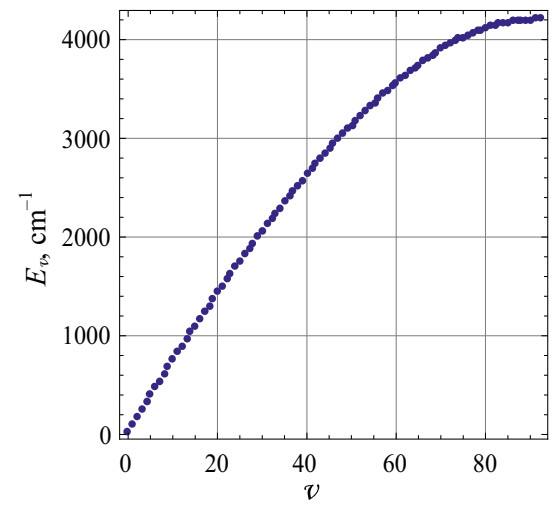

$a$

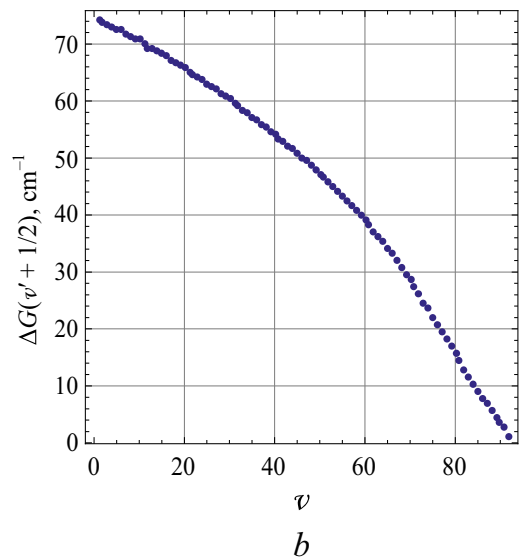

Fig. 4. Present $a b$ initio vibrational energies $E_{v}$ (a) and vibrational intervals $\Delta G\left(v^{\prime}+1 / 2\right)$ (b) for the ground state $X^{1} \Sigma^{+}$of the ${ }^{39} K^{85} \mathrm{Rb}$ molecule. 

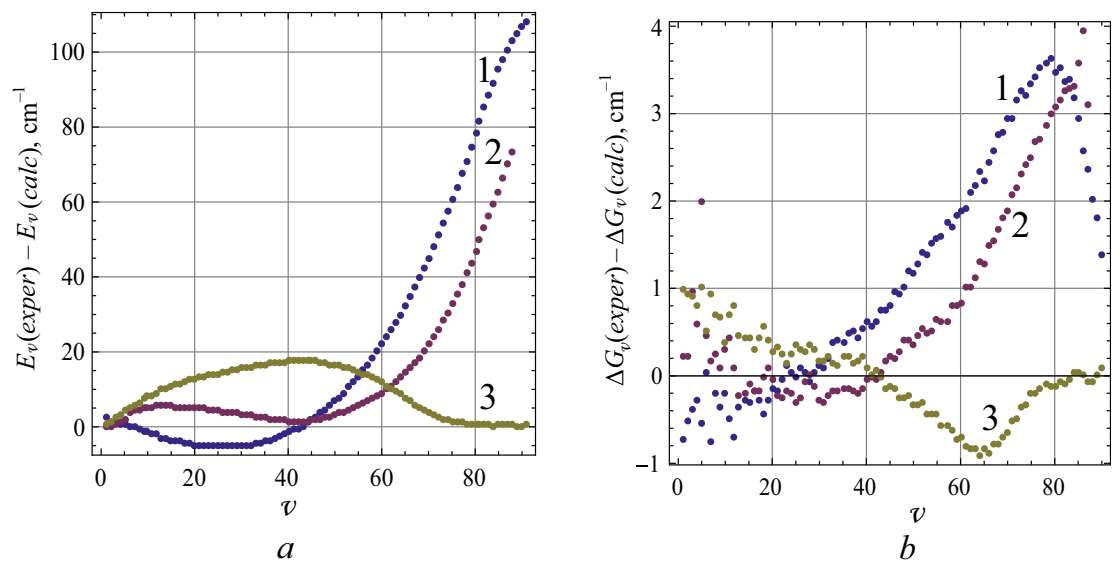

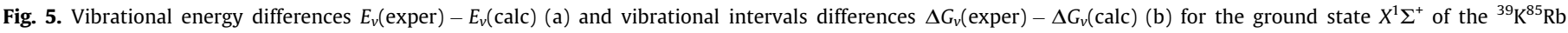
molecule: (1) Rousseau et al. [32], (2) Chen et al. [41], (3) present work.

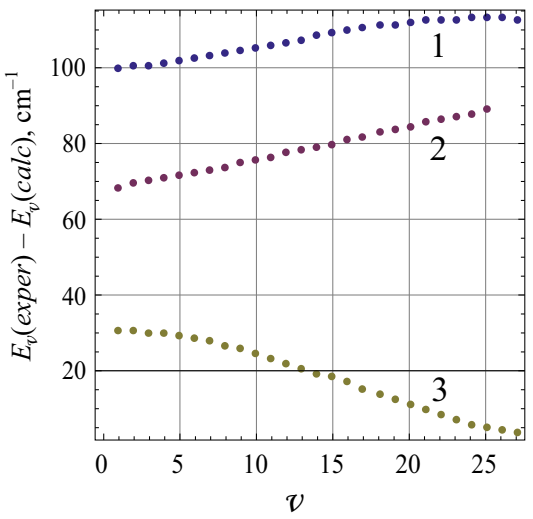

$a$

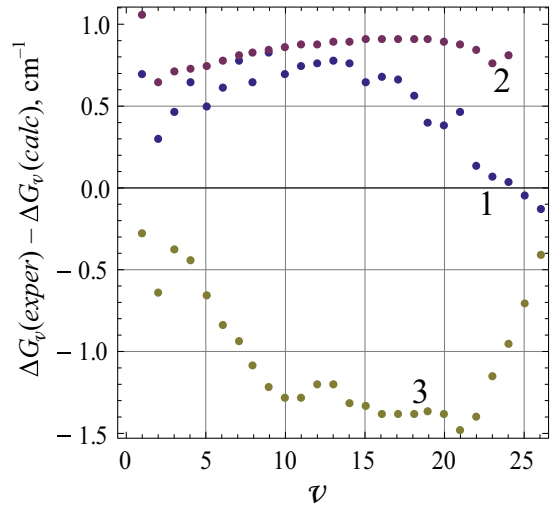

$b$

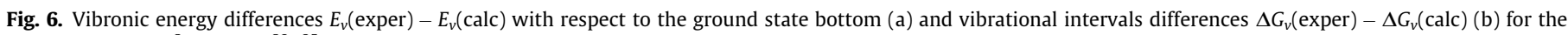
first triplet state $1^{3} \Sigma^{+}$of the ${ }^{39} \mathrm{~K}^{85} \mathrm{Rb}$ molecule: (1) Rousseau et al. [32], (2) Chen et al. [41], (3) present work.
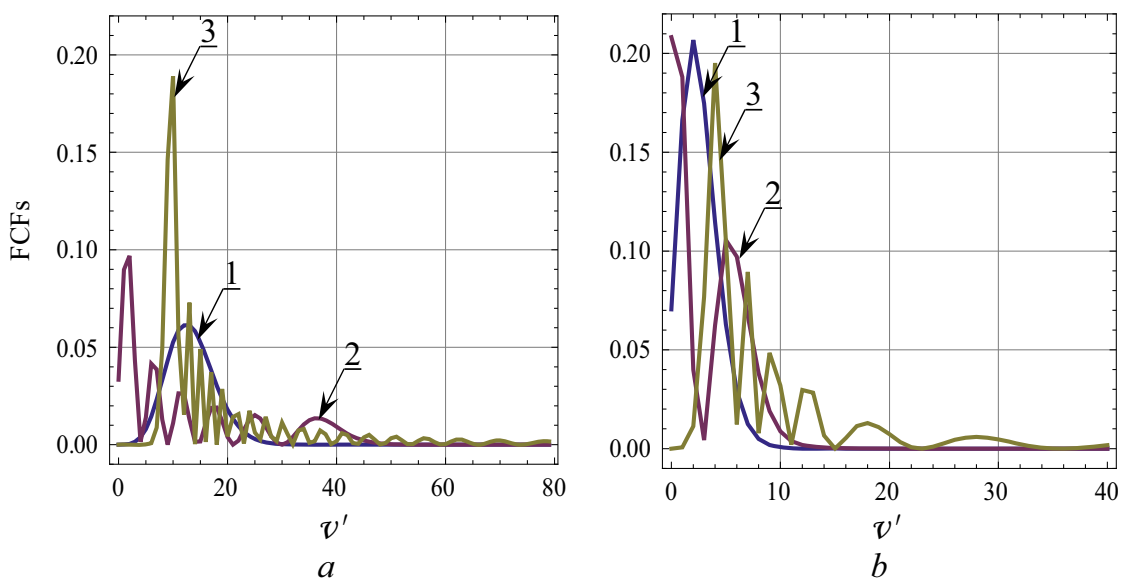

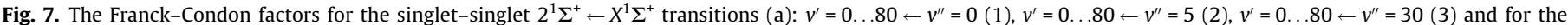
$1^{1} \Pi \leftarrow X^{1} \Sigma^{+}$transitions $(b): v^{\prime}=0 \ldots 40 \leftarrow v^{\prime \prime}=0(1), v^{\prime}=0 \ldots 40 \leftarrow v^{\prime \prime}=2(2), v^{\prime}=0 \ldots 40 \leftarrow v^{\prime \prime}=11$ (3) calculated with $\Delta J=0$ of the ${ }^{39} \mathrm{~K}^{85} \mathrm{Rb}$ molecule.

and 10.50-10.55 ̊, respectively. These “jumps" may take place either because of a failure of the convergence criterion of calculations at the CASSCF or MRCI level of theory or due to inclusion into the SA-procedure of an extraneous state and is obviously connected with the automatic scanning of the PEC. We performed a calculation similar to Chen et al. [41] using a quantum chemical package MOLPRO [52,53] and found that the CASSCF calculations of the PEC near $4.3 \AA$ have not been brought to the required level of convergence. This resulted in an error in MRCI calculations and further in the distortion of the PEC's shape (see curve 2 in Fig. 2). 

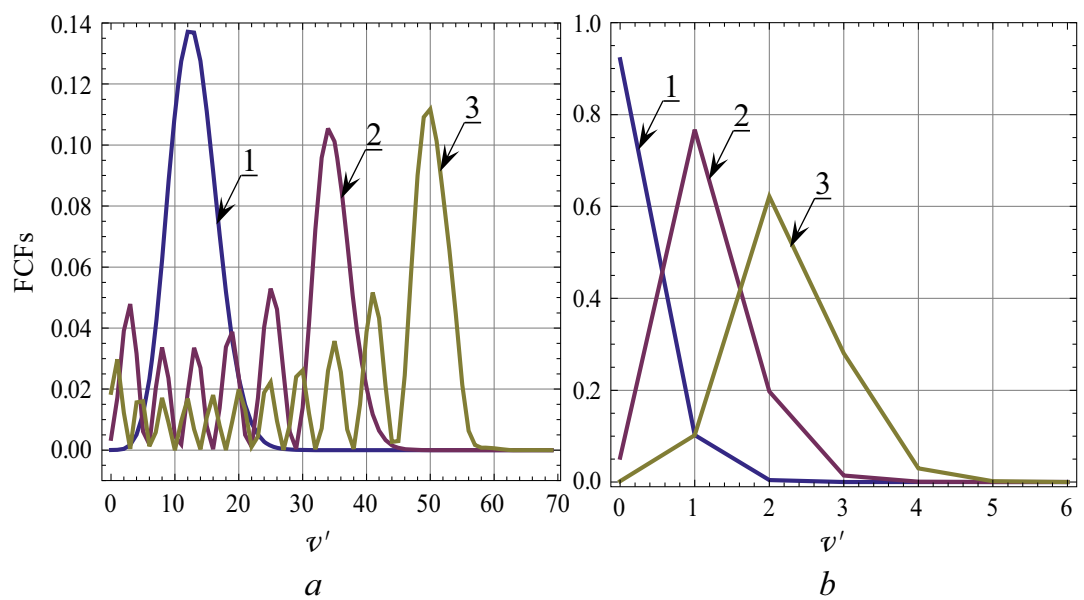

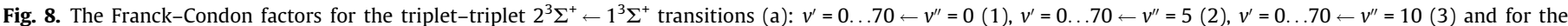
singlet-triplet $1^{3} \Pi \leftarrow X^{1} \Sigma^{+}$transitions (b): $v^{\prime}=0 \ldots 6 \leftarrow v^{\prime \prime}=0(1), v^{\prime}=0 \ldots 6 \leftarrow v^{\prime \prime}=1(2), v^{\prime}=0 \ldots 6 \leftarrow v^{\prime \prime}=6$ (3) calculated with $\Delta J=0$ of the ${ }^{39} \mathrm{~K}^{85} \mathrm{Rb}$ molecule.

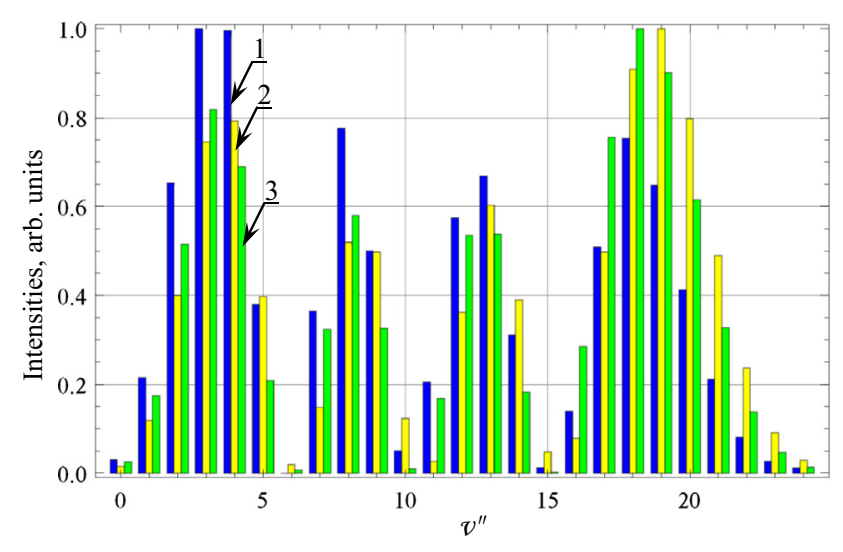

Fig. 9. Experimental (1) and calculated without shift (2) and with a shift of the $2^{1} \Sigma^{+}$ term by $-0.02 \AA$ (3) relative intensity distributions for the $2^{1} \Sigma^{+}\left(v^{\prime}=3, J^{\prime}=26\right) \rightarrow$ $X^{1} \Sigma^{+}\left(v^{\prime \prime}=0 \ldots 24, J^{\prime \prime}=25,27\right)$ transitions of the ${ }^{39} \mathrm{~K}^{85} \mathrm{Rb}$ molecule.

The present calculated molecular constants for the first triplet state $1^{3} \Sigma^{+}$(see Table 1 ) also agree rather well with experimental data of Pashov et al. [13]. The energy differences between the empirical and calculated curves for the first triplet state are shown in Fig. 3. Note that here the energy differences are also presented with respect to the bottom of the potential well of the ground singlet state. These data also demonstrate a successful reproduction of the first triplet state PEC by present calculations. The "breaks" and "ejections" in the curves in the region of 4.2-5.2 ̊ are supposingly caused by the above-mentioned reason since here the empirical functions are matched at the point $4.965 \AA$.

\subsection{Vibrational states}

Hereafter for the singlet, triplet and spin-mixed PECs the vibrational energies were calculated up to the dissociation limit of the respective state. The results for the ground state of the ${ }^{39} \mathrm{~K}^{85} \mathrm{Rb}$ molecule are presented in Fig. 4 and compared with experimental [13] and previous theoretical studies [32,41] in Fig. 5. One can see from Fig. 5a that the difference between experimental and present calculated vibrational energies for all vibrational levels of the ${ }^{39} \mathrm{~K}^{85} \mathrm{Rb}$ molecule ground state does not exceed $20 \mathrm{~cm}^{-1}$. These data demonstrate that present calculations (see curve 3 ) reproduce the system of vibrational states of the ground electronic term of the ${ }^{39} \mathrm{~K}^{85} \mathrm{Rb}$ molecule better than the other ones (see curves 1 and 2). As can be seen from Fig. 5b, the present calculated vibrational intervals (see curve 3 ) describe the experiment with the accuracy within $\pm 1 \mathrm{~cm}^{-1}$ in the entire $v$ range. It should be noted that the deviation from the experiment at large $v$ observed in Fig. $5 \mathrm{a}$ and $\mathrm{b}$ for curves 1 and 2 is connected with the smaller $D_{e}$ value in $[32,41]$.

For the first triplet state $1^{3} \Sigma^{+}$of the ${ }^{39} \mathrm{~K}^{85} \mathrm{Rb}$ molecule the calculated vibronic energies also well correlate with the experimental ones [13] (Fig. 6a). The deviations of the calculated vibrational intervals $\Delta G\left(v^{\prime}+1 / 2\right)$ from the experimental values of [13] do not exceed $1.5 \mathrm{~cm}^{-1}$ (Fig. 6b). Note that the energies in Fig. 6a refer to the bottom of the singlet ground electronic state.

The similar analysis was performed for the $1^{1} \Pi$ state with the experimental data of Kim et al. [3] and theoretical data of Rousseau et al. [32] (see Figs. S3 and S4 in the supplementary material). The calculated vibrational energies and vibrational intervals for other $\left(2^{1} \Sigma^{+}, 3^{1} \Sigma^{+}, 2^{1} \Pi, 1^{3} \Pi\right.$ and $\left.2^{3} \Sigma^{+}\right)$states (excluding quasibound states $2^{3} \Pi_{\Omega}$ and $3^{3} \Sigma^{+}$) are given in the supplementary material in Figs. S1, S2 and S5-S7.

\subsection{Franck-Condon factors}

Basing on $a b$ initio PECs and calculated vibrational eigenfunctions we predicted the Franck-Condon factors (FCFs) between the relevant states of the ${ }^{39} \mathrm{~K}^{85} \mathrm{Rb}$ molecule. The FCFs have been calculated for the singlet-singlet, triplet-triplet and singlet-triplet rovibronic transitions. These calculations were performed for transitions between the fixed vibrational level $v^{\prime \prime}$ of the lower electronic state and the sequence of vibrational levels $v^{\prime}$ of the upper electronic state (applying the selection rules $\Delta J=0$ or $\Delta J= \pm 1$ ). The FCFs for the singlet-singlet $2^{1} \Sigma^{+} \leftarrow X^{1} \Sigma^{+}$and $1^{1} \Pi \leftarrow X^{1} \Sigma^{+}$transitions with $\Delta J=0$ are shown in Fig. 7 . The equilibrium internuclear distances for $X^{1} \Sigma^{+}$and $2^{1} \Sigma^{+}$states are markedly different, while the ones for $X^{1} \Sigma^{+}$and $1^{1} \Pi$ terms are more close to each other (see Table 1 and Fig. 1). This is reflected in the FCFs distributions. For the $1^{1} \Pi \leftarrow X^{1} \Sigma^{+}$transitions the nonzero FCFs have a narrower distribution, with larger values, than for the $2^{1} \Sigma^{+} \leftarrow X^{1} \Sigma^{+}$transitions. The FCFs for the triplet-triplet $2^{3} \Sigma^{+} \leftarrow 1^{3} \Sigma^{+}$transitions and for the intersystem crossing singlet-triplet $1^{3} \Pi \leftarrow X^{1} \Sigma^{+}$transitions with $\Delta J=0$ are shown in Fig. 8. The equilibrium internuclear distances of the $X^{1} \Sigma^{+}$and $1^{3} \Pi$ states are practically coinciding (see Table 1), therefore the FCF between the $X^{1} \Sigma^{+}\left(v^{\prime \prime}=0\right)$ level and the $1^{3} \Pi\left(v^{\prime}=0\right)$ level is close to $1(0.9204)$. This value well agrees 
with Kobayashi et al. [15] data (0.9474). The FCF between the $X^{1} \Sigma^{+}$ $\left(v^{\prime \prime}=1\right)$ level and the $1^{3} \Pi\left(v^{\prime}=0\right)$ level $(0.0533)$ also agrees with Kobayashi et al. [15] value (0.0500).

The calculated, assuming constant transition dipole moment, normalized fluorescence intensities (FCFs multiplied by the forth degree of the transitions frequencies) of the $P, R$ doublet progression $2^{1} \Sigma^{+}\left(v^{\prime}=3, J^{\prime}=26\right) \rightarrow X^{1} \Sigma^{+}\left(v^{\prime \prime}=0 \ldots .24, J^{\prime \prime}=25,27\right)$ transitions (second and third sets, or yellow and green ${ }^{1}$ bars in Fig. 9) are compared with the experimental normalized intensities, measured in the Laser Centre, University of Latvia (first set, or blue bars in Fig. 9). KRb molecules were produced in a linear heat pipe at $310^{\circ} \mathrm{C}$. The experimental intensity distribution in the given progression was obtained from the laser induced fluorescence spectrum, which was recorded by Fourier Transform spectrometer IFS-125HR (Bruker Optics) with $0.03 \mathrm{~cm}^{-1}$ spectral resolution. The transition $2^{1} \Sigma^{+} \quad\left(v^{\prime}=3\right.$, $\left.J^{\prime}=26\right) \leftarrow X^{1} \Sigma^{+}\left(v^{\prime \prime}=2, J^{\prime \prime}=25\right)$ was excited with Titan Sapphire laser at $10908.902 \mathrm{~cm}^{-1}$ frequency. The experimental line intensities were determined for both doublet components separately and then averaged, since $P$ - and $R$-line intensities coincided within few percents. Measured intensities were corrected for spectral sensitivity of the InGaAs detector. More details can be obtained from Ref. [54].

The second data set (yellow bars) in Fig. 9 is obtained from the present $a b$ initio ground $X^{1} \Sigma^{+}$and $a b$ initio excited $2^{1} \Sigma^{+}$state PECs without any shifts. For the third data set (green bars) the $a b$ initio PEC of the $2^{1} \Sigma^{+}$term was shifted by $-0.02 \AA$. We note that the intensities calculated from the shifted PEC are closer to the observed intensities distribution than the non-shifted one. Consequently, the equilibrium internuclear distance of the $2^{1} \Sigma^{+}$PEC might be less than the one predicted by calculations. Taking into account that the $2^{1} \Sigma^{+}$term is in fact partially mixed with the $1^{3} \Pi_{0}$ term $\left(95 \% 2^{1} \Sigma^{+}\right.$and $\left.5 \% 1^{3} \Pi_{0}\right)$ [54], and, what is more, that the transition dipole moment function [37] is ignored here, the calculated FCFs data satisfactory reflect the experimentally observed intensity distribution.

\section{Conclusions}

We have calculated PECs for the ground and some low-lying excited states of the KRb molecule at the CASSCF(2,14)/XMCQDPT2 level of theory. The $a b$ initio results (PECs, molecular spectroscopic constants, vibrational energies, vibrational intervals, FCFs) obtained without any empirical fitting are in good agreement with the experimental and previous theoretical data. The calculated parameters of the ground $X^{1} \Sigma^{+}$state are in the best agreement among all previously performed for $\mathrm{KRb}$ molecule $a b$ initio calculations.

We assume that the calculations at the CASSCF/XMCQDPT2 level of theory are well applicable for electronic structure calculations of other heavy alkali diatomic molecules, especially high accuracy is expected for the ground electronic state.

\section{Acknowledgments}

This work has been supported by the State Committee on Science and Technology of the Republic of Belarus (Nr. F14LAT060) and Latvian-Belarusian cooperation project by Ministry of Education and Science of the Republic of Latvia (Nr. LVBY/2015/3). The authors are grateful to Prof. Chuan-Lu Yang (Ludong University, China), Dr. Alexandra Freidzon (Photochemistry Center, Russia) and Dr. Alexander A. Granovsky, Firefly Project, Russia) for many helpful discussions.

${ }^{1}$ For interpretation of color in Fig. 9, the reader is referred to the web version of this article.

\section{Appendix A. Supplementary material}

Supplementary data associated with this article can be found, in the online version, at http://dx.doi.org/10.1016/j.comptc.2016.04. 029.

\section{References}

[1] K.-K. Ni, S. Ospelkaus, M.H.G. de Miranda, A. Pe'er, B. Neyenhuis, J.J. Zirbel, S. Kotochigova, P.S. Julienne, D.S. Jin, J. Ye, A high phase-space-density gas of polar molecules, Science 322 (2008) 231-235.

[2] J.-T. Kim, Y. Lee, B. Kim, D. Wang, W.C. Stwalley, P.L. Gould, E.E. Eyler, Spectroscopic prescription for optimal stimulated Raman transfer of ultracold heteronuclear molecules to the lowest rovibronic level, Phys. Rev. A 84 (2011) 062511-1-062511-7.

[3] J.-T. Kim, Y. Lee, B. Kim, D. Wang, W.C. Stwalley, P.L. Gould, E.E. Eyler, Spectroscopic analysis of the coupled $1^{1} \Pi, 2^{3} \Sigma^{+}\left(\Omega=0^{-}, 1\right)$, and $b^{3} \Pi\left(\Omega=0^{ \pm}, 1\right.$, 2 ) states of the KRb molecule using both ultracold molecules and molecular beam experiments, Phys. Chem. Chem. Phys. 13 (2011) 18755-18761.

[4] J.-T. Kim, Y. Lee, B. Kim, D. Wang, P.L. Gould, E.E. Eyler, W.C. Stwalley, Spectroscopic investigation of the A and $3{ }^{1} \Sigma^{+}$states of ${ }^{39} \mathrm{~K}^{85} \mathrm{Rb}$, J. Chem. Phys. 137 (2012) 244301-1-244301-8

[5] D. Borsalino, B. Londoño-Florèz, R. Vexiau, O. Dulieu, N. Bouloufa-Maafa, E. Luc-Koenig, Efficient optical schemes to create ultracold $\mathrm{KRb}$ molecules in their rovibronic ground state, Phys. Rev. A 90 (2014) 033413-1-033413-14.

[6] A.J. Ross, C. Effantin, P. Croset, E. Boursey, The ground state of KRb from laserinduced fluorescence, J. Phys. B: At. Mol. Phys. 23 (1990) L247-L252.

[7] S. Kasahara, C. Fujiwara, N. Okada, H. Katô, M. Baba, Doppler-free opticaloptical double resonance polarization spectroscopy of the ${ }^{39} \mathrm{~K}^{85} \mathrm{Rb} 1{ }^{1} \Pi$ and $2^{1} \Pi$ states, J. Chem. Phys. 111 (1999) 8857-8866.

[8] C. Amiot, J. Vergès, J. d'Incan, C. Effantin, The $(3)^{1} \Pi-(3)^{1} \Sigma^{+}$system of KRb, Chem. Phys. Lett. 315 (1999) 55-60.

[9] C. Amiot, J. Vergès, The KRb ground electronic state potential up to $10 \AA$, J. Chem. Phys. 112 (2000) 7068-7074.

[10] C. Amiot, J. Vergès, C. Effantin, J. d'Incan, The KRb (2) ${ }^{1} \Pi$ electronic state, Chem. Phys. Lett. 321 (2000) 21-24.

[11] C. Amiot, The KRb $(2)^{3} \Sigma^{+}$electronic State, J. Mol. Spectrosc. 203 (2000) 126131.

[12] Y. Lee, C. Yun, Y. Yoon, T. Kim, B. Kim, The $530 \mathrm{~nm}$ system of KRb observed in a pulsed molecular beam: new electric quadrupole transitions $\left(1^{1} \Delta-X^{1} \Sigma^{+}\right)$, J. Chem. Phys. 115 (2001) 7413-7419.

[13] A. Pashov, O. Docenko, M. Tamanis, R. Ferber, H. Knöckel, E. Tiemann, Coupling of the $X^{1} \Sigma^{+}$and $a^{3} \Sigma^{+}$states of KRb, Phys. Rev. A 76 (2007) 022511-1-02251110.

[14] J. Banerjee, D. Rahmlow, R. Carollo, M. Bells, E. Eyler, P. Gould, C. Stwalley, Spectroscopy and applications of the $3^{3} \Sigma^{+}$electronic state of ${ }^{39} \mathrm{~K}^{85} \mathrm{Rb}$, J. Chem. Phys. 139 (2013) 174316-1-174316-9.

[15] J. Kobayashi, K. Aikawa, K. Oasa, S. Inouye, Prospects for narrow-line cooling of KRb molecules in the rovibrational ground state, Phys. Rev. A 89 (2014) 021401-1-021401-5.

[16] N. Okada, S. Kasahara, T. Ebi, M. Baba, H. Katô, Optical-optical double resonance polarization spectroscopy of the $B^{1} \Pi$ state of ${ }^{39} \mathrm{~K}^{85} \mathrm{Rb}$, J. Chem. Phys. 105 (1996) 3458-3465.

[17] Y. Lee, Y. Yoon, B. Kim, L. Li, S. Lee, Observation of the $3^{3} \Sigma^{+}-X^{1} \Sigma^{+}$transition of $\mathrm{KRb}$ by resonance enhanced two-photon ionization in a pulsed molecular beam: hyperfine structures of ${ }^{39} \mathrm{~K}^{85} \mathrm{Rb}$ and ${ }^{39} \mathrm{~K}^{87} \mathrm{Rb}$ isotopomers, J. Chem. Phys. 120 (2004) 6551-6556.

[18] D. Wang, J. Qi, M.F. Stone, O. Nikolayeva, H. Wang, B. Hattaway, S.D. Gensemer, P.L. Gould, E.E. Eyler, W.C. Stwalley, Photoassociative production and trapping of ultracold KRb molecules, Phys. Rev. Lett. 93 (2004) 243005-1-243005-4.

[19] D. Wang, J. Qi, M.F. Stone, O. Nikolayeva, B. Hattaway, S.D. Gensemer, H. Wang, W.T. Zemke, P.L. Gould, E.E. Eyler, W.C. Stwalley, The photoassociative spectroscopy, photoassociative molecule formation, and trapping of ultracold ${ }^{39} \mathrm{~K}^{85} \mathrm{Rb}$, Eur. Phys. J. D 31 (2004) 165-178.

[20] W.C. Stwalley, Improved dissociation energy of the ${ }^{39} \mathrm{~K}^{85} \mathrm{Rb}$ molecule, J. Chem. Phys. 122 (2005) 084319-1-084319-3.

[21] D. Wang, E.E. Eyler, P.L. Gould, W.C. Stwalley, Spectra of ultracold KRb molecules in near-dissociation vibrational levels, J. Phys. B: At. Mol. Opt. Phys. 39 (2006) S849-S856.

[22] D. Wang, J.T. Kim, C. Ashbaugh, E.E. Eyler, P.L. Gould, W.C. Stwalley, Rotationally resolved depletion spectroscopy of ultracold $\mathrm{KRb}$ molecules, Phys. Rev. A 75 (2007) 032511-1-032511-5.

[23] J.T. Kim, D. Wang, E.E. Eyler, P.L. Gould, W.C. Stwalley, Spectroscopy of ${ }^{39} \mathrm{~K}^{85} \mathrm{Rb}$ triplet excited states using ultracold $a^{3} \Sigma^{+}$state molecules formed by photoassociation, New J. Phys. 11 (2009) 055020-1-055020-20.

[24] K. Aikawa, D. Akamatsu, J. Kobayashi, M. Ueda, T. Kishimoto, S. Inouye, Toward the production of quantum degenerate bosonic polar molecules, ${ }^{41} \mathrm{~K}^{87} \mathrm{Rb}$, New J. Phys. 11 (2009) 055035-1-055035-12.

[25] Y. Lee, Y. Yoon, A. Muhammad, J.-T. Kim, S. Lee, B. Kim, The 480 nm system of KRb: $1^{3} \Delta_{1}, 4^{1} \Sigma^{+}$, and $5^{1} \Sigma^{+}$states, J. Phys. Chem. A 114 (2010) 7742-7748.

[26] K. Aikawa, D. Akamatsu, M. Hayashi, J. Kobayashi, M. Ueda, S. Inouye, Predicting and verifying transition strengths from weakly bound molecules, Phys. Rev. A 83 (2011) 042706-1-042706-7. 
[27] Y. Lee, Y. Yoon, J.-T. Kim, S. Lee, B. Kim, Unravelling complex spectra of a simple molecule: REMPI study of the $420 \mathrm{~nm}$ band system of KRb, ChemPhysChem 12 (2011) 2018-2023.

[28] J. Banerjee, D. Rahmlow, R. Carollo, M. Bellos, E.E. Eyler, P.L. Gould, W.C. Stwalley, Direct photoassociative formation of ultracold KRb molecules in the lowest vibrational levels of the electronic ground state, Phys. Rev. A 86 (2012) 053428-1-053428-5.

[29] J. Banerjee, D. Rahmlow, R. Carollo, M. Bellos, E.E. Eyler, P.L. Gould, W.C. Stwalley, Spectroscopy of the double minimum $3^{3} \Pi_{\Omega}$ electronic state of ${ }^{39} \mathrm{~K}^{85} \mathrm{Rb}, \mathrm{J}$. Chem. Phys. 138 (2013) 164302-1-164302-8.

[30] T. Leininger, G.-H. Jeung, Calculation of the weakly coupled 1 and $2^{1} \Pi$ twin states of KRb, Phys. Rev. A 51 (1995) 1929-1935.

[31] A. Yiannopoulou, T. Leininger, A.M. Lyyra, G.-H. Jeung, Theoretically calculated rovibronic transition spectra of KRb, Int. J. Quantum Chem. 57 (1996) 575-585.

[32] S. Rousseau, A.R. Allouche, M. Aubert-Frécon, Theoretical study of the electronic structure of the KRb molecule, J. Mol. Spectrosc. 203 (2000) 235243.

[33] S.J. Park, Y.J. Choi, Y.S. Lee, G.-H. Jeung, Ab initio calculations of the electronic states of KRb, Chem. Phys. 257 (2000) 135-145.

[34] S. Kotochigova, P.S. Julienne, E. Tiesinga, Ab initio calculation of the KRb dipole moments, Phys. Rev. A 68 (2003) 022501-1-022501-7.

[35] S. Kotochigova, E. Tiesinga, P.S. Julienne, Photoassociative formation of ultracold polar KRb molecules, Eur. Phys. J. D 31 (2004) 189-194.

[36] A. Aymar, O. Dulieu, Calculation of accurate permanent dipole moments of the lowest ${ }^{1,3} \Sigma^{+}$states of heteronuclear alkali dimers using extended basis sets, J. Chem. Phys. 122 (2005) 204302-1-204302-9.

[37] R. Beuc, M. Movre, T. Ban, G. Pichler, M. Aymar, O. Dulieu, W.E. Ernst, Predictions for the observation of KRb spectra under cold conditions, J. Phys. B: At. Mol. Opt. Phys. 39 (2006) S1191-S1202.

[38] S. Kotochigova, E. Tiesinga, P.S. Julienne, Multi-channel modelling of the formation of vibrationally cold polar KRb molecules, New J. Phys. 11 (2009) 055043-1-055043-15.

[39] W.C. Stwalley, J. Banerjee, M. Bellos, R. Carollo, M. Recore, M. Mastroianni, Resonant coupling in the heteronuclear alkali dimers for direct photoassociative formation of $\mathrm{X}(0,0)$ ultracold molecules, J. Phys. Chem. A 114 (2010) 81-86.

[40] E.R. Meyer, J.L. Bohn, Product-state control of bi-alkali-metal chemical reactions, Phys. Rev. A 82 (2010) 042707-1-042707-6.

[41] K. Chen, C.-L. Yang, M.-S. Wang, X.-G. Ma, W.-W. Liu, Analytical potential energy functions and spectroscopic properties for the ground and low-lying excited states of KRb, Spectrochim. Acta A 99 (2012) 57-61.

[42] W.C. Stwalley, M. Bellos, R. Carollo, J. Banerjee, M. Bermudez, Shortcuts for understanding rovibronic spectroscopy of ultracold alkali metal diatomic molecules, Mol. Phys. 110 (2012) 1739-1755.
[43] P. Łobacz, P. Jasik, J.E. Sienkiewicz, Theoretical study of highly-excited states of KRb molecule, Cent. Eur. J. Phys. 11 (2013) 1107-1114.

[44] D.A. Fedorov, A. Derevianko, S.A. Varganov, Accurate potential energy, dipole moment curves, and lifetimes of vibrational states of heteronuclear alkali dimers, J. Chem. Phys. 140 (2014) 184315-1-184315-9.

[45] A.A. Granovsky, Extended multi-configuration quasi-degenerate perturbation theory: the new approach to multi-state multi-reference perturbation theory, J. Chem. Phys. 134 (2011) 214113-1-214113-15.

[46] I.S. Lim, P. Schwerdtfeger, B. Metz, H. Stoll, All-electron and relativistic pseudopotential studies for the group 1 element polarizabilities from $\mathrm{K}$ to element 119, J. Chem. Phys. 122 (2005) 104103-1-104103-13.

[47] S.-W. Chang H.A. Witek, Choice of optimal shift parameter for the intruder state removal techniques in Multireference Perturbation Theory, J. Chem. Theory Comput. 8 (2012) 4053-4061.

48] M.B. Shundalau, A.A. Minko, Determination of the optimal energy denominator shift parameter of KRb electronic states in quantum chemical computations using perturbation theory, J. Appl. Spectrosc. 82 (2016) 901904.

[49] Alex A. Granovsky, Firefly version 8, <http://classic.chem.msu.su/gran/firefly/ index.html> (accessed 10.03.16)

[50] G.A. Pitsevich, A.E. Malevich, V. Sablinskas, I.U. Doroshenko, V.E. Pogorelov, V. Balevicius, Vibrational analysis of hydroxyl group in the pyridine $\mathrm{N}$-oxide trichloroacetic acid complex using the anharmanic approximation and computations of 1D and 2D potential surfaces, J. Spectrosc. Dyn. 3 (2013) 19-1-19-11.

[51] G.A Pitsevich, A.E Malevih, Simple method of the formation of the Hamiltonian matrix for some Schrodinger equations describing the molecules with large amplitude motions, Opt. Photonics J. 2 (2012) 332-337.

[52] H.-J. Werner, P.J. Knowles, G. Knizia, F.R. Manby, M. Schutz, Molpro: a generalpurpose quantum chemistry program package, WIREs Comput. Mol. Sci. 2 (2012) 242-253.

[53] H.-J. Werner, P.J. Knowles, G. Knizia, F.R. Manby, M. Schutz, P. Celani, T. Korona, R. Lindh, A. Mitrushenkov, G. Rauhut, K.R. Shamasundar, T.B. Adler, R.D. Amos, A. Bernhardsson, A. Berning, D.L. Cooper, M.J.O. Deegan, A.J. Dobbyn, F. Eckert, E. Goll, C. Hampel, A. Hesselmann, G. Hetzer, T. Hrenar, G. Jansen, C. Koppl, Y. Liu, A.W. Lloyd, R.A. Mata, A.J. May, S.J. McNicholas, W. Meyer, M.E. Mura, A Nicklass, D.P. O’Neill, P. Palmieri, D. Peng, K. Pfluger, R. Pitzer, M. Reiher, T. Shiozaki, H. Stoll, A.J. Stone, R. Tarroni, T. Thorsteinsson, M. Wang, MOLPRO version 2012.1, a package of ab initio programs, see <http://www.molpro.net>

[54] K. Alps, A. Kruzins, M. Tamanis, R. Ferber, E.A. Pazyuk, A.V. Stolyarov, Fouriertransform spectroscopy and deperturbation analysis of the spin-orbit coupled $A^{1} \Sigma^{+}$and $b^{3}$ II states of KRb, J. Chem. Phys. 144 (2016) 144310-1-144310-14. 